\title{
A influência de calçados no arco longitudinal medial do pé e na cinemática dos membros inferiores de crianças no início da fase de aquisição de marcha*
}

\section{The Influence of the Shoe over the Medial Foot Arch and the Lower Limbs Kinematics in Toddlers}

\author{
Liria Akie Okai-Nobrega ${ }^{1,2(1)}$ Thiago Ribeiro Teles Santos ${ }^{10}$ Ana Paula Lage ${ }^{20}$

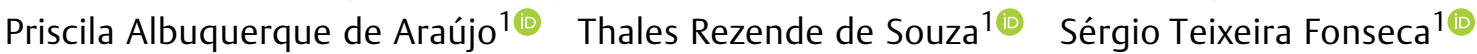

${ }^{1}$ Departamento de Fisioterapia, Universidade Federal de Minas Gerais, Belo Horizonte, MG, Brasil

2 Anamê Baby Design, Belo Horizonte, MG, Brasil
Endereço para correspondência Liria Akie Okai- Nóbrega, PhD, Departmento de Fisioterapia, Av. Presidente Antônio Carlos, 6627, Belo Horizonte, MG, Brasil (e-mail: okainobrega@gmail.com).

Rev Bras Ortop 2022;57(1):167-174.

\section{Resumo \\ Objetivo Avaliar o comportamento do arco longitudinal medial do pé (ALM) e os parâmetros cinemáticos dos membros inferiores durante a deambulação com calçados biomiméticos (CBs) e não biomiméticos (NB1, NB2, NB3 e NB4) em crianças no início da fase de aquisição da marcha. \\ Métodos Foram avaliadas quatro crianças no início da fase de aquisição da marcha nas seguintes condições: andar descalço, andar com CBs e calçados NB1, NB2, NB3 e NB4 em solo plano. O calçado biomimético é descrito como biomimético por emular pisos naturais e irregulares por meio de uma palmilha interna dinâmica. O ALM e a cinemática do quadril, joelho e tornozelo durante a marcha foram avaliados por meio de sistema de análise do movimento tridimensional. A similaridade entre as curvas \\ Palavras-chave \\ - marcha \\ - criança \\ - biomimética \\ - calçados cinemáticas das condições descalça e com calçado foi analisada por meio do cálculo de root mean square error (RMSE). \\ Resultados OCB foi o que apresentou maior magnitude do ALM e maior diferença do ALM em relação à condição descalça (maior RMSE). O CB apresentou ainda menor diferença na cinemática das articulações do joelho e tornozelo durante a marcha}

Trabalho desenvolvido no Laboratório de Análise do Movimento (LAM) do Departamento de Fisioterapia, Universidade Federal de Minas Gerais, Belo Horizonte, MG, Brasil. recebido

10 de Maio de 2021

aceito

09 de Setembro de 2021

Publicado on-line

de Janeiro 24, 2022
DOI https://doi.org/

10.1055/s-0041-1741021. ISSN 0102-3616. (c) 2022. Sociedade Brasileira de Ortopedia e Traumatologia. All rights reserved.

This is an open access article published by Thieme under the terms of the Creative Commons Attribution-NonDerivative-NonCommercial-License, permitting copying and reproduction so long as the original work is given appropriate credit. Contents may not be used for commercial purposes, or adapted, remixed, transformed or built upon. (https://creativecommons.org/ licenses/by-nc-nd/4.0/)

Thieme Revinter Publicações Ltda., Rua do Matoso 170, Rio de Janeiro, RJ, CEP 20270-135, Brazil 
quando comparado à condição descalça (menor RMSE). O calçado NB2 apresentou a cinemática do quadril mais semelhante à condição descalça (menor RMSE).

Conclusão Os calçados CB e NB2 que apresentam a região do antepé mais larga geraram menores diferenças na cinemática dos membros inferiores. Além disso, o ALM foi maior no $C B$ provavelmente devido a seu design ser diferente daquele dos demais calçados.

Abstract

Keywords

- gait

- child

- biomimetics

- shoes
Objective To evaluate the biomechanical behavior of the medial longitudinal arch (MLA) of the foot and the kinematic parameters of the lower limbs with biomimetic footwear (BF) and non-biomimetic (NB1, NB2, NB3 and NB4) footwear in children at the beginning of the gait acquisition phase.

Methods Four toddlers were evaluated at the beginning of the gait acquisition phase under the following conditions: walking barefoot, ambulation with BF and NB1, NB2, NB3 and NB4 footwear in hard floor. BF is described as biomimetic because of its property of emulating natural and irregular floors through a dynamic internal insole. The MLA and kinematics of the hip, knee, and ankle during gait were evaluated by three-dimensional motion analysis system. The similarity between the kinematic curves of barefoot and footwear conditions was analyzed by root mean square error (RMSE).

Results The use of BF presented the highest magnitude of MLA and the greatest difference in relation to barefoot condition (higher RMSE). The BF showed less difference in the kinematics of the knee and ankle joints during gait when compared to barefoot condition (lower RMSE). NB2 footwear presented hip kinematics more similar to those of barefoot condition (lower RMSE).

Conclusion Biomimetics footwear and NB2 shoes (both with wider forefoot region) generated smaller differences in lower limbs compared to barefoot. In addition, the MLA was higher in the BF, probably because different design from that of the other shoes.

\section{Introdução}

A vida urbana faz com que crianças fiquem constantemente calçadas. $^{1-3}$ Durante a locomoção, o calçado afeta diretamente a forma como as forças de reação do solo são geradas e transmitidas para todo o corpo, ${ }^{4,5}$ uma vez que é a interface entre o corpo e o solo. Apesar do crescente interesse na literatura sobre o efeito do calçado na locomoção, esse efeito nas crianças, ainda em fase de desenvolvimento da marcha tem sido pouco explorado. 6

$\mathrm{O}$ início da marcha independente acontece em torno dos 15 meses de idade. ${ }^{7,8} 0$ pé possui como principais funções na marcha: i) acomodar irregularidade do solo e manutenção do equilíbrio; ii) suporte de peso e absorção de carga e iii) gerar o movimento para frente, transmitindo forças propulsivas. ${ }^{9}$ Dessa forma, durante a locomoção, a criança precisa mover sua massa corporal à frente ao mesmo tempo em que se equilibra em apoio uni e bipodal, trazendo um duplo desafio de estabilidade e progressão. ${ }^{10}$

No início da aquisição da marcha independente, os ossos do pé possuem vários centros de ossificação conectados por tecidos moles, ${ }^{11}$ e o desenvolvimento das estruturas ósseas acontece até os cinco anos de idade. ${ }^{12}$ Esse processo de ossificação ao longo do crescimento corrobora com a ausência do arco longitudinal medial (ALM) do pé em crianças no início da aquisição da marcha. ${ }^{9}$ Assim, é esperado que a própria marcha venha a promover o desenvolvimento do ALM.

Além de proteger os pés, o tamanho, a forma e o design de calçados podem interferir no desenvolvimento estrutural e na sua função e, assim, gerar impactos sobre o sistema musculoesquelético a longo prazo. ${ }^{3}$ Estudos indicam que o uso de calçados na primeira infância pode levar a alterações morfológicas nos pés de indivíduos, em comparação com pessoas que não usam calçados. ${ }^{13,14}$ Essas alterações no formato do pé podem reduzir a habilidade de atenuar o impacto das forças pelas estruturas podais, alterando até a forma como se caminha. Estudos têm destacado a importância do andar descalço no desenvolvimento e função do pé. $^{13,14} \mathrm{O}$ andar descalço proporciona força muscular, mobilidade podal, evita deformidades estáticas e afeta a altura do ALM. ${ }^{2,13,14}$ O caminhar em diferentes tipos naturais de terreno tem o potencial de afetar a mecânica e a energética da locomoção, ${ }^{15}$ além de gerar constantes ajustes para manter a estabilidade. ${ }^{16}$ Dessa forma, o calçado usado por crianças durante a fase de desenvolvimento da marcha pode 
ter um papel importante sobre o desenvolvimento do sistema musculoesquelético.

O design de produtos inspirados na natureza tem sido chamado de biomimetismo. ${ }^{17}$ Considerando os efeitos do andar descalço proporciona experiências tanto em solos planos como em terrenos naturais, calçados biomiméticos poderiam impactar no ALM de crianças na fase de desenvolvimento da marcha e na cinemática das articulações do membro inferior durante a marcha. $O$ objetivo deste estudo foi avaliar o comportamento do ALM e dos parâmetros cinemáticos dos membros inferiores de crianças durante a marcha usando calçados biomiméticos (CBs) e não biomiméticos, comparando-os com o ato de andar descalço em solo plano.

\section{Métodos}

Um estudo de $\operatorname{casos}^{18}$ foi realizado com 4 crianças com idade entre 13 e 17 meses $(15,50 \pm 1,73$ meses), com massa corporal de 10 a $12 \mathrm{~kg}(11,20 \pm 0,74 \mathrm{~kg})$ e altura de 78 a $90 \mathrm{~cm}$ $(82 \pm 5 \mathrm{~cm})$, com desenvolvimento motor típico. Todas as crianças tinham entre 2 e 3 meses de experiência de caminhar sem apoio. Este estudo foi aprovado pelo Comitê de Ética em Pesquisa da instituição (parecer número 2.083.328), e todos os pais assinaram o termo de assentimento livre e esclarecido antes de qualquer procedimento ser realizado com as crianças.

As crianças realizaram marcha independente em solo plano e foram avaliadas em seis condições, cuja sequência foi estabelecida por tabela de aleatorização: 1) Descalço; 2) CB; 3) Calçado não biomimético 1 (NB1); 4) Calçado não biomimético 2 (NB2); 5) Calçado não biomimético 3 (NB3); 6) Calçado não biomimético 4 (NB4). A - Tabela 1 traz as características e a - Figura 1, as imagens dos calçados testados.

A avaliação da marcha foi realizada por meio de sistema de análise tridimensional do movimento (Qualisys Medical $A B$, Gothenburg, Suécia). Pelve, coxa, perna e pé foram rastreados durante a marcha por meio de 22 marcadores retro refletivos (-Figura 2). Marcadores anatômicos foram usados para a calibração estática a fim de definir os segmentos corporais e os respectivos sistemas de coordenadas.

Os dados foram analisados no software Visual3D (C-Motion Inc., Rockville, MD, EUA). Todos os dados foram filtrados com filtro Butterworth passa baixa de quarta ordem em frequência de corte de $6 \mathrm{~Hz}$. Os centros articulares foram estimados conforme literatura. ${ }^{19}$ Os seguintes ângulos no plano sagital foram extraídos: quadril, joelho e tornozelo. O ALM do pé foi determinado pelo ângulo entre o vetor que conecta o marcador do calcâneo e do navicular e o vetor que conecta o marcador do navicular e o da cabeça do primeiro metatarso. ${ }^{20}$ Dessa forma, quanto menor o ângulo, maior o ALM do pé. Além disso, os membros inferiores foram classificados como membro de apoio e membro de avanço. ${ }^{10}$ Nessa classificação, o membro inferior com maior comprimento da passada é classificado como o de avanço, e o contralateral como o de apoio. ${ }^{10}$

As crianças caminharam livremente, em velocidade auto selecionada, e estímulos verbais, visuais e lúdicos foram utilizados para facilitar a tarefa. Foram analisados no mínimo 10 passadas completas para cada membro inferior.

Para estimar a semelhança entre as curvas da variação angular em relação ao tempo de cada um dos calçados e o caminhar descalço, foi utilizado o índice root mean square error (RMSE). Esse índice é sempre positivo e mede a magnitude da diferença das curvas cinemáticas. ${ }^{20}$ Em outras palavras, esse escore mede a diferença entre os dados da curva cinemática de cada condição (CB, NB1, NB2, NB3, NB4) em relação à média de um padrão de referência, que neste estudo foi a condição descalça. Assim, quanto maior o valor do RMSE, maior a diferença entre as curvas obtidas com os calçados analisados em relação a andar descalço.

\section{Resultados}

Todos os participantes apresentaram maior comprimento da passada no lado direito $(0,399 \pm 0,08 \mathrm{~m})$ do que no lado esquerdo $(0,393 \pm 0,08 \mathrm{~m})$, que foram classificados como membro inferior de avanço e de apoio, respectivamente.

As curvas médias do ALM nas condições avaliadas estão apresentadas na - Figura 3, e os valores de RMSE estão representados na - Figura 4. As - Figuras 3a e $\mathbf{3 b}$ apresentam a curva média do ALM durante a fase de apoio do ciclo de marcha na condição descalça. Nessas curvas, é possível observar que o ângulo do ALM é pequeno no contato inicial da marcha e, gradualmente, aumenta, sendo que o pico desse ângulo ocorre em torno dos $80 \%$ da fase de apoio, provavelmente, contribuindo com a impulsão do corpo à frente. Observa-se ainda que, na condição descalça, o pé do membro inferior de avanço (-Figura $\mathbf{3 b}$ ) apresentou um maior ângulo do ALM durante a fase de apoio que o do membro de apoio (-Figura 3a). As -Figuras 3c e 3d apresentam as curvas do ALM tanto na condição descalça (linha vermelha), usada como referência, quanto com os diferentes calçados investigados. Observa-se que o efeito do

Tabela 1 Medidas dos calçados utilizados

\begin{tabular}{|l|l|l|l|l|l|l|}
\hline Calçado & $\begin{array}{l}\text { Comprimento } \\
(\mathbf{c m})\end{array}$ & Massa (g) & $\begin{array}{l}\text { Largura externa } \\
\text { antepé }(\mathbf{c m})\end{array}$ & $\begin{array}{l}\text { Largura interna } \\
\text { antepé }(\mathrm{cm})\end{array}$ & $\begin{array}{l}\text { Palmilha } \\
\text { interna }\end{array}$ & $\begin{array}{l}\text { Distância do } \\
\text { pé ao solo (cm) }\end{array}$ \\
\hline Biomimético (CB) & 13,6 & 517,0 & 7,3 & 6,7 & Dinâmica & 1,0 \\
\hline Não biomimético 1 (NB1) & 14,6 & 291,0 & 6,9 & 6,0 & Estática & 2,0 \\
\hline Não biomimético 2 (NB2) & 14,2 & 195,5 & 6,9 & 6,4 & Estática & 2,0 \\
\hline Não biomimético 3 (NB3) & 14,1 & 429,5 & 6,5 & 5,6 & Estática & 1,5 \\
\hline Não biomimético 4 (NB4) & 13,9 & 561,5 & 6,8 & 5,6 & Estática & 1,3 \\
\hline
\end{tabular}

Fonte: Elaborado pelos autores (2021). 

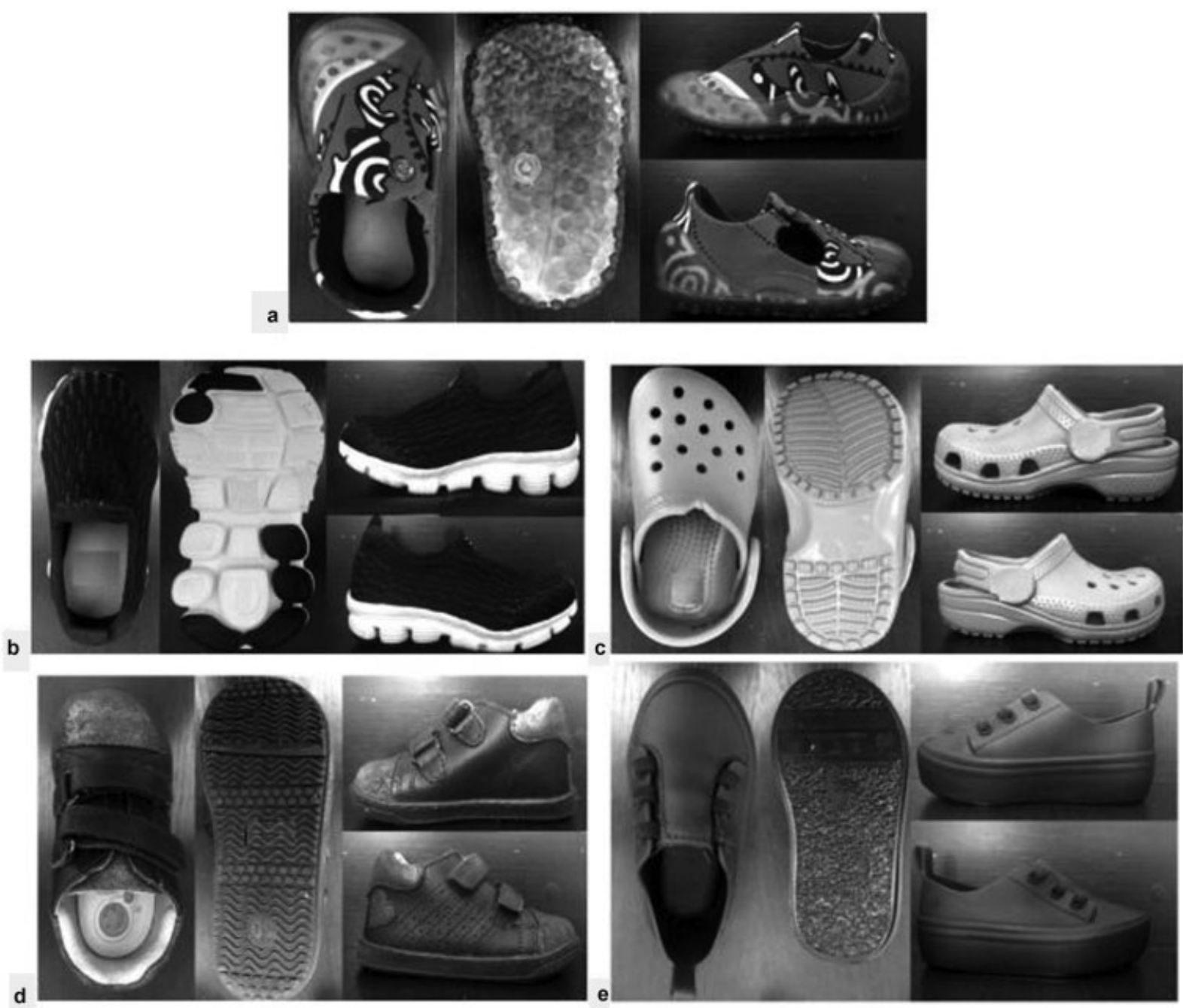

Fig. 1 Imagens dos calçados infantis avaliados. (a) CB: calçado biomimético, (b) NB1: calçado não biomimético 1; (c) NB2: calçado não biomimético 2; (d) NB3: calçado não biomimético 3, (e) NB4: calçado não biomimético 4. Fonte: Elaborado pelos autores (2021).

calçado é diferente dependendo da função do pé: membro inferior de apoio (-Figura 3c) ou avanço (-Figura 3d). O CB (linha azul turquesa) foi o calçado que apresentou o maior ALM (i.e., menor ângulo) em ambos os pés. Esse achado se relaciona com o maior RMSE (- Figura 4). Ou seja, o CB foi o calçado que apresentou o ALM mais diferente da condição descalça.

As curvas médias do quadril, joelho e tornozelo em cada condição estão apresentadas na - Figura 5. Observa-se que as maiores diferenças entre as condições estão no final da fase de apoio (aproximadamente entre 50 e $70 \%$ do ciclo de marcha) na articulação do tornozelo. As diferenças em RMSE entre as condições calçada e com os calçados investigados estão representadas na - Figura 6. Os calçados que apresentaram menor RMSE (ou seja, maior similaridade com o andar descalço) foram o CB (coluna azul turquesa) e NB2 (coluna verde) (-Figura 6).

\section{Discussão}

Em linhas gerais, este estudo descreveu que os diferentes calçados analisados resultaram em algum grau de dife- rença em relação à condição descalça, o que sugere que o calçado pode afetar o padrão de movimento das crianças. Além disso, os achados indicaram que a deambulação com $\mathrm{CB}$ foi, em geral, mais similar ao andar descalço nas articulações do membro inferior (i.e., menor RMSE) e mais diferente quanto ao ALM do pé (i.e., maior RMSE). Assim, o design do calçado parece influenciar as variáveis cinemáticas do membro inferior de crianças durante o início da aquisição da marcha. Este estudo sugere que um design que considera o biomimetismo impacta no ALM das crianças nessa fase além de permitir movimentos articulares de membros inferiores mais semelhantes ao andar descalço.

Existe evidência de crescente aumento no interesse em calçados para crianças, principalmente nos últimos 10 anos. ${ }^{5}$ Apesar disso, há poucas pesquisas sobre o impacto do calçado em crianças, principalmente na fase de aquisição da marcha. Alguns autores $^{3,21}$ afirmam que o calçado pode ter um efeito a longo prazo na função do pé. Um calçado com um design biomimético e anatômico é capaz de proteger o pé e pode também manter a flexibilidade natural, largura do antepé e sustentar o ALM do pé. 


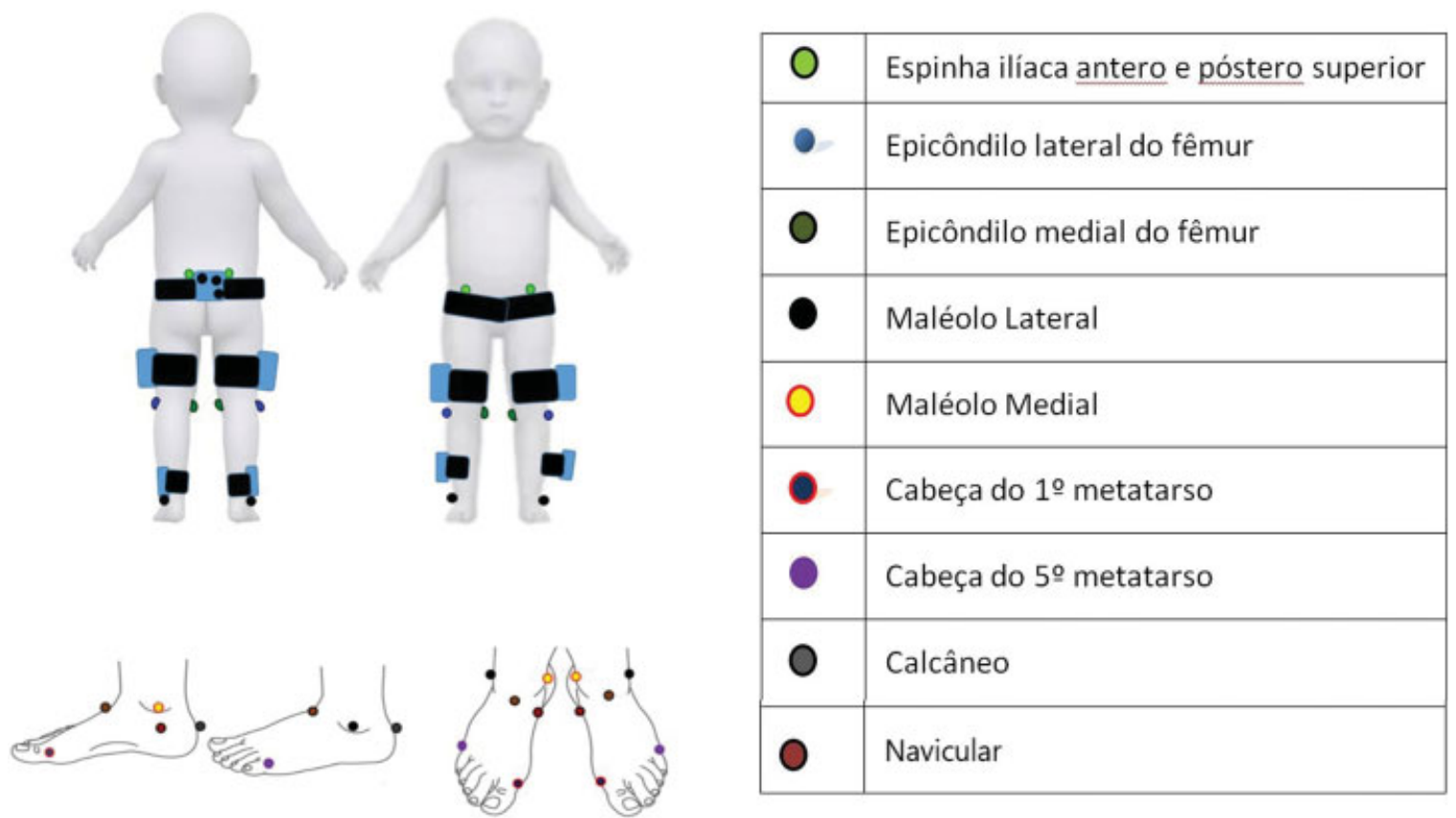

Fig. 2 Desenho esquemático que ilustra o posicionamento dos marcadores retro refletivos no corpo das crianças. A imagem inferior ilustra de forma mais detalhada a posição dos marcadores nos pés. Fonte: Elaborado pelos autores (2021).
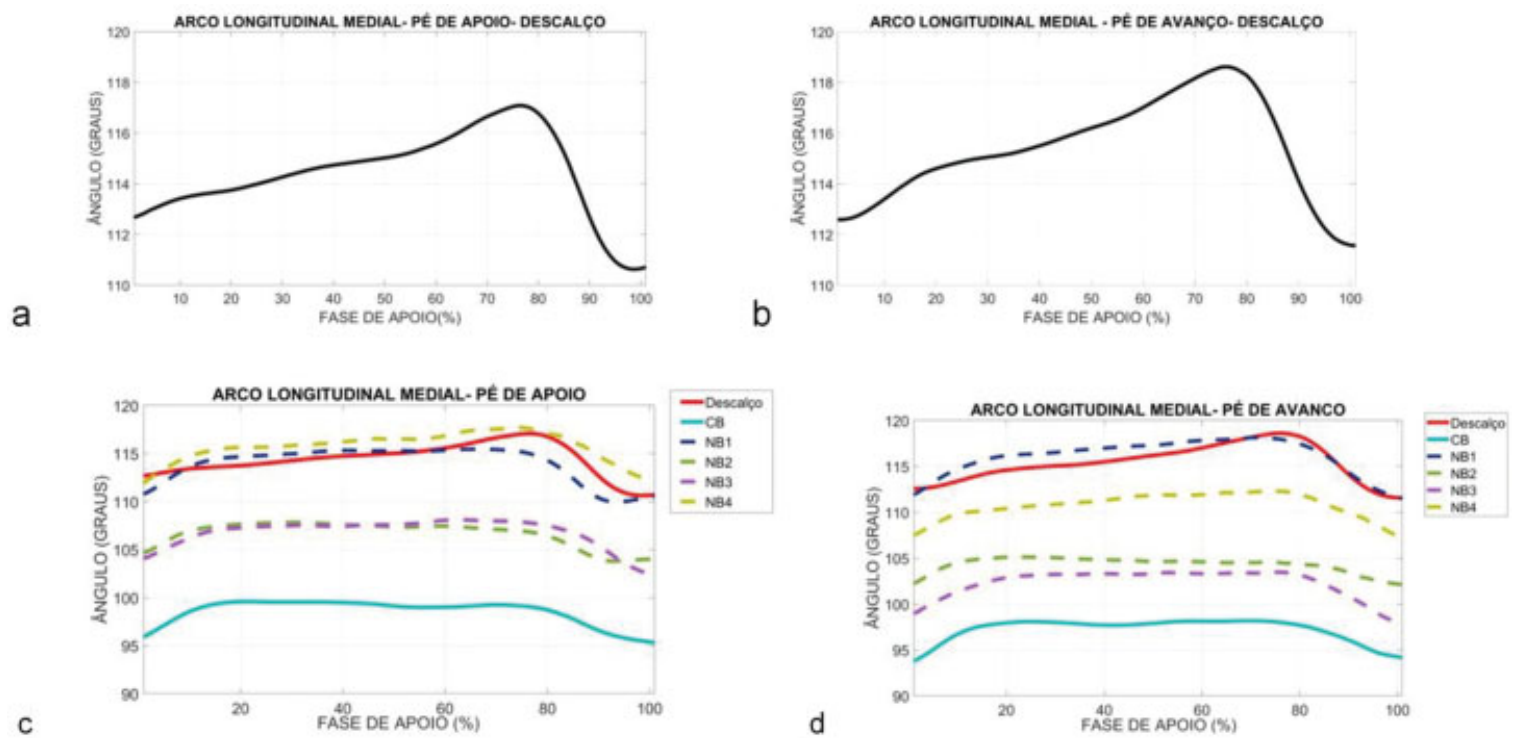

Fig. 3 Gráficos da média das curvas arco longitudinal medial (ALM) do pé durante a marcha de quatro crianças em todas as condições avaliadas. (a) Descalço pé de apoio, (b) Descalço pé de avanço, (c) Pé de apoio: CB (calçado biomimético), NB1 (calçado não biomimético 1), NB2 (calçado não biomimético 2), NB3 (calçado não biomimético 3), NB4 (calçado não biomimético 4) e, (d) Pé de avanço: CB (calçado biomimético), NB1 (calçado não biomimético 1), NB2 (calçado não biomimético 2), NB3 (calçado não biomimético 3), NB4 (calçado não biomimético 4). Fonte: Elaborado pelos autores (2021).

O ALM é dependente de estruturas anatômicas passivas e ativas. A aponeurose plantar é considerada uma estrutura importante durante a fase de apoio, juntamente com os ligamentos, enquanto os músculos da perna e os intrínsecos do pé atuam como um suporte ativo. ${ }^{22} \mathrm{~A}$ - Figura 3 traz a média das curvas do ALM do pé durante a marcha das quatro crianças na condição descalça. Graficamente, apesar de o ALM ainda estar em desenvolvimento, na condição descalça, o comportamento das curvas é muito semelhante às curvas do
ALM de adultos. ${ }^{22,23}$ As diferenças descritas pelo RMSE relativas ao ALM, principalmente do CB com o andar descalço, podem ser explicadas por meio de duas conjecturas. A primeira seria por meio de um efeito mecânico da palmilha do $\mathrm{CB}$. Durante a fase de apoio da marcha, uma pressão maior é aplicada na parte lateral do pé. O CB avaliado possui uma palmilha dinâmica que contém material similar a grãos de areia. Dessa forma, esse material provavelmente se acumula sob a região com menor pressão (ou seja, a região medial do pé), 

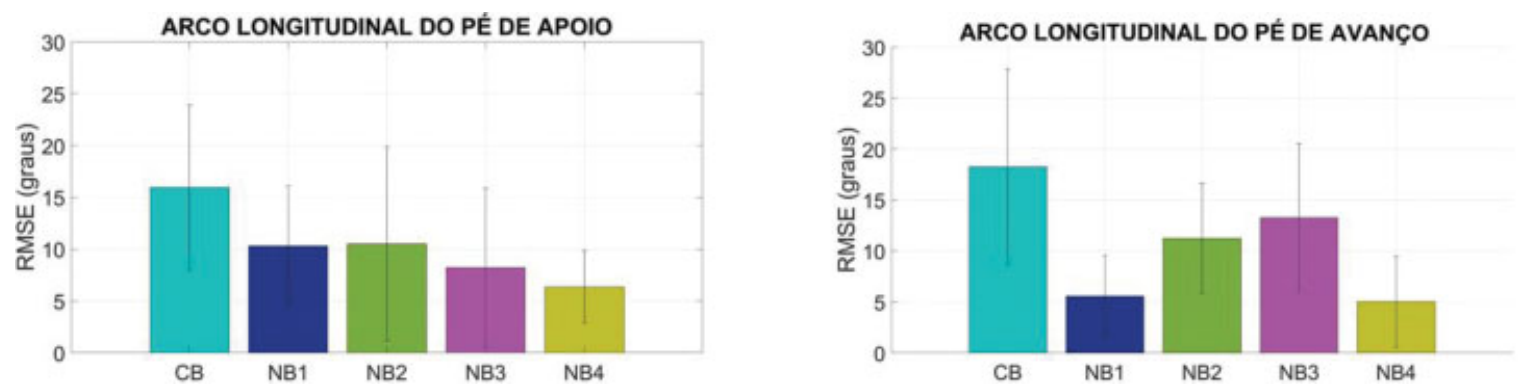

Fig. 4 Gráficos das médias das diferenças das curvas do arco longitudinal do pé (RMSE) durante a marcha de quatro crianças com diferentes calçados, comparados com a condição descalça. CB (calçado biomimético), NB1 (calçado não biomimético 1), NB2 (calçado não biomimético 2), NB3 (calçado não biomimético 3), NB4 (calçado não biomimético 4). Fonte: Elaborado pelos autores (2021).

a
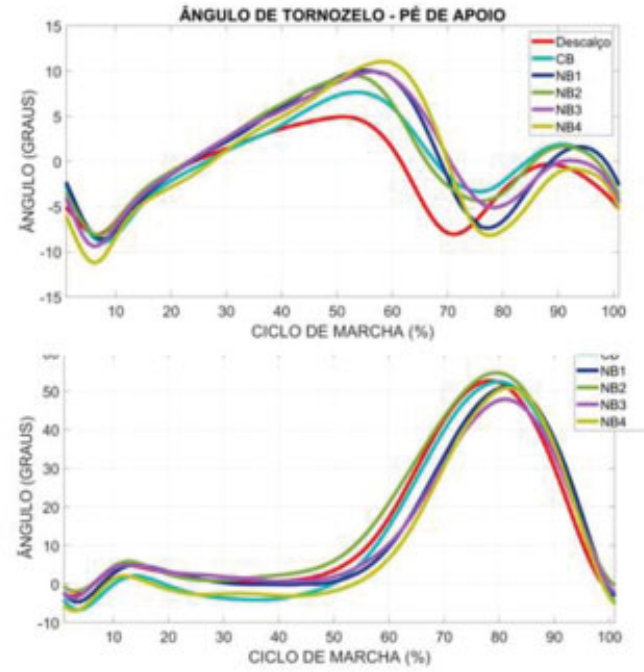

C

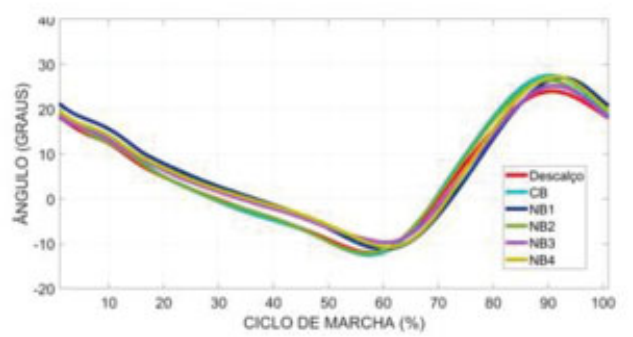

e
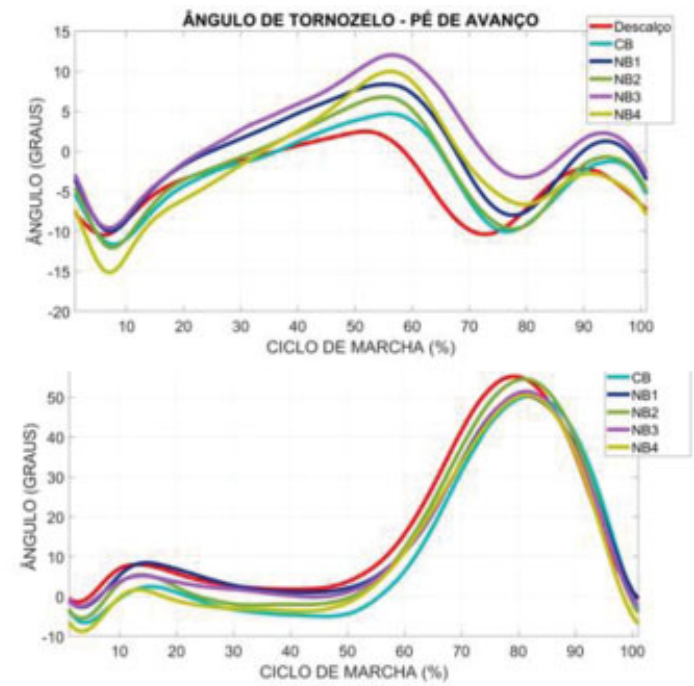

d

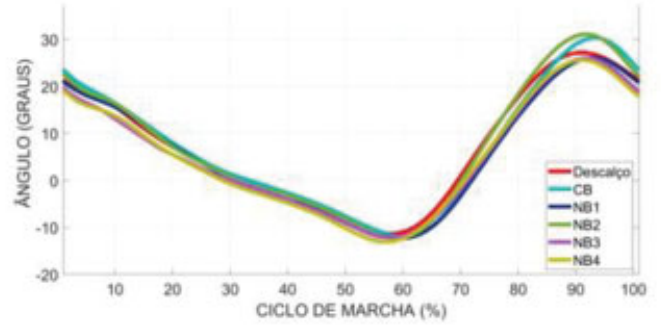

f

Fig. 5 Gráficos da média das curvas articulares do tornozelo (a e b), joelho (c e d) e quadril (e ef) durante a marcha de quatro crianças em todas as condições avaliadas: Descalço, CB (calçado biomimético), NB1 (calçado não biomimético 1), NB2 (calçado não biomimético 2), NB3 (calçado não biomimético 3), NB4 (calçado não biomimético 4). Dorsiflexão e flexão: valores positivos nos gráficos. Fonte: elaborado pelos autores (2021).

favorecendo assim o aumento do ALM. A segunda conjectura é que a palmilha dinâmica do $\mathrm{CB}$ pode ter estimulado a atividade dos músculos da região plantar do pé. Nessa perspectiva, a contração muscular favoreceu a formação do ALM. Essa segunda possibilidade corrobora as observações de Kung et al., ${ }^{1}$ que afirmaram que o andar descalço aumentaria a força muscular na região do tornozelo e pé, especificamente nos flexores plantares, inversores e adutores do tornozelo. A maior diferença do ALM do pé, da condição $\mathrm{CB}$ em relação à condição descalça, descrita pelo RMSE, juntamente com o padrão observado dessa variável nos gráficos descritivos sugerem que o $\mathrm{CB}$ promoveu um aumento da rigidez do mediopé. Esse efeito corrobora o papel do pé em atuar como uma alavanca rígida no final da fase de apoio para promover a propulsão do corpo à frente durante a marcha. ${ }^{24}$ Ressalta-se que a literatura indica que o ALM não é influenciado somente por fatores locais. 0 seu desenvolvimento é dependente de vários fatores, como o peso corporal, atividade física, etnicidade e até mesmo a idade. ${ }^{12,25}$

A natureza da morfologia e anatomia do pé do bebê, no início da aquisição de marcha, tem sido associada às dimensões do corpo dessa criança. ${ }^{26} \mathrm{O}$ peso do corpo mais que dobra desde o nascimento até seu primeiro ano de vida. Além disso, 0 comprimento do membro inferior aumenta em torno de $50 \%$ do nascimento até os 18 meses de idade, e $50 \%$ do comprimento do pé quando adulto é alcançado em torno de 12 a 18 meses de idade. ${ }^{26}$ Estudo $^{27}$ indica que o desenvolvimento 

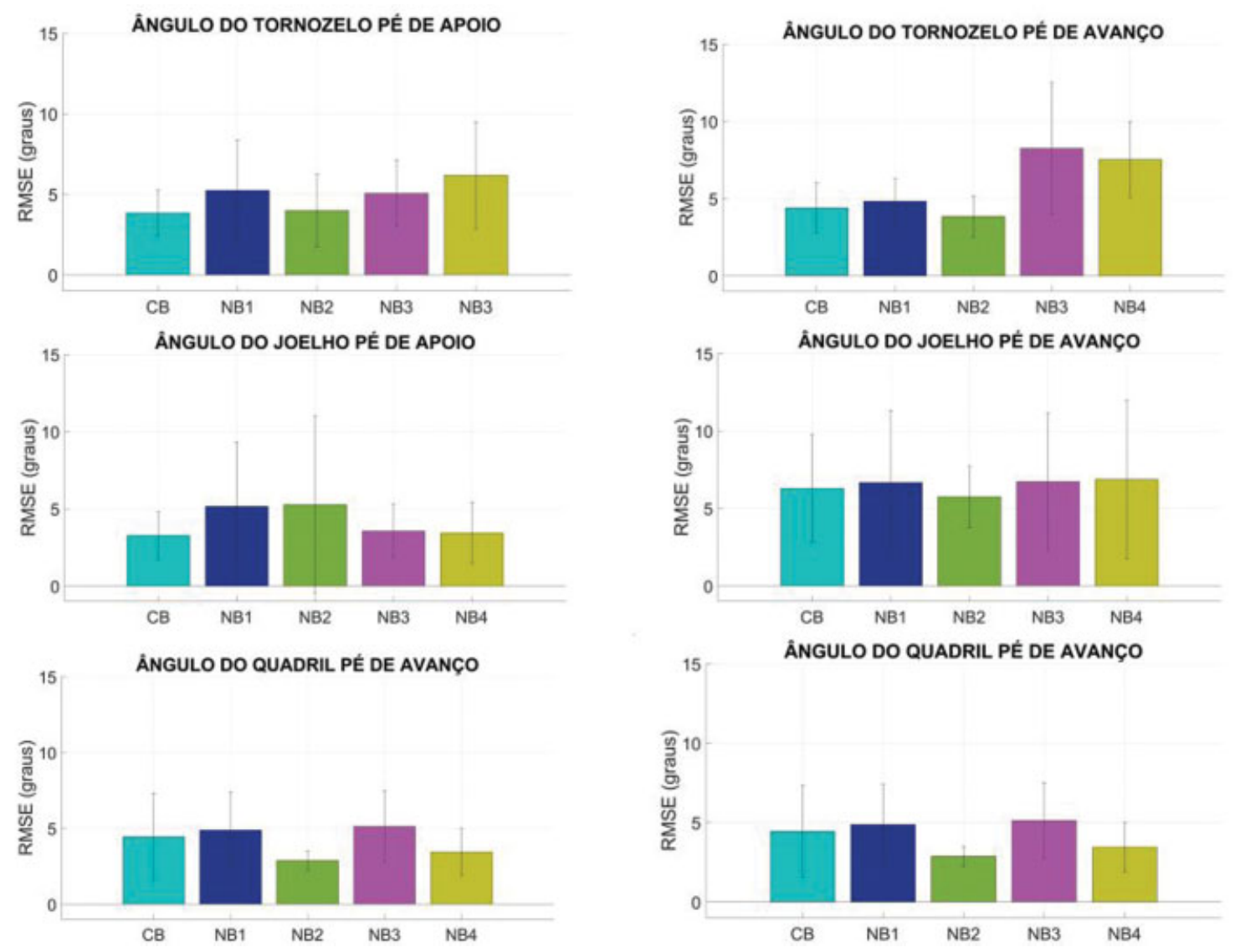

Fig. 6 Gráficos das médias das diferenças das curvas das variações angulares das articulações do tornozelo, joelho e quadril (RMSE) durante a marcha de quatro crianças com diferentes calçados comparados com a condição descalça. CB (calçado biomimético), NB1 (calçado não biomimético 1), NB2 (calçado não biomimético 2), NB3 (calçado não biomimético 3), NB4 (calçado não biomimético 4). Fonte: Elaborado pelos autores (2021).

motor depende não somente da maturação neuromuscular, mas, também da confluência de outros fatores externos que afetam diretamente, como, por exemplo, a postura (gravidade e posicionamentos), crescimento físico (massa, comprimento e proporções), força muscular e o desenvolvimento do equilíbrio. A idade entre dois e cinco anos de idade é considerado um período em que os estímulos resultantes do andar impactam significativamente para o desenvolvimento do pé. ${ }^{26} \mathrm{Um}$ calçado que mimetiza a irregularidade de superfícies como o andar na areia pode fornecer uma variação ambiental de forma segura para a criança. Crianças urbanas, geralmente, são expostas a superfícies mais planas e regulares no seu dia a dia. Dessa forma, calçados que mimetizam um ambiente natural, como o $\mathrm{CB}$, podem oferecer diferentes estímulos e assim, auxiliar no desenvolvimento adequado do sistema musculoesquelético, de forma segura para a vida urbana. Entretanto, deve se considerar que este é um estudo transversal e assim, não podemos concluir sobre os efeitos de médio e longo prazo. Futuros estudos são necessários para investigar os impactos no desenvolvimento musculoesquelético do uso de calçados que mimetizam solos irregulares.

Todos os calçados apresentaram alguma diferença descrita pelo RMSE com a condição descalça tanto localmente no tornozelo quanto em articulações proximais. Os calçados que apresentaram, em geral, menor diferença foram $C B$ e NB2. Esses calçados são similares no design relativo à largura interna do antepé, em que apresentam maior largura interna comparado aos demais calçados (-Tabela 1). Provavelmente, esses calçados não restringem (ou restringem menos) o aumento da largura do antepé esperada durante a descarga de peso. $^{28}$ Durante a descarga de peso, espera-se que a pronação do pé favoreça a absorção da carga e permita a adaptação do pé ao solo. ${ }^{29}$ Especificamente, durante a pronação, espera-se que à medida em que o tálus aduz em relação ao calcâneo, ele se desloque anteriormente (i.e., flexão plantar do tálus em relação ao calcâneo). ${ }^{29}$ Esse movimento é acompanhado por deslocamento anterior e inferior do navicular e cuneiformes, juntamente com um deslocamento anterior dos três raios (i.e., metatarso e suas respectivas falanges) mediais do antepé em relação aos outros dois raios laterais do antepé. ${ }^{29}$ Esse movimento é revertido durante a supinação do pé, em que o pé se torna uma alavanca rígida para favorecer a propulsão do corpo à frente. ${ }^{29} \mathrm{O}$ estudo de Thelen demonstra que os movimentos do pé se acoplam aos movimentos do joelho e quadril no plano sagital. ${ }^{30}$ Assim, provavelmente, calçados que restrinjam minimamente o movimento do antepé favorecem que os acoplamentos articulares do membro inferior no plano sagital sejam mais semelhantes ao andar descalço. $\mathrm{O}$ impacto da largura do calçado no antepé foi indicado por Franklin et al., ${ }^{28}$ que indicaram que o uso de calçados por toda a vida tem resultado em alterações funcionais e anatômicas, principalmente, em relação a uma largura de antepé reduzida. 
A generalização dos achados deste estudo é limitada, uma vez que este é um estudo descritivo baseado na análise de apenas quatro crianças. Além disso, os efeitos descritos neste estudo foram imediatos, ou seja, as crianças não tinham experiência prévia com nenhum dos modelos dos calçados avaliados. Os achados descritos neste estudo de caso podem contribuir com o planejamento de futuros estudos para avaliar os efeitos agudo e crônico do uso de calçados com diferentes designs.

\section{Conclusão}

A descrição analisada neste trabalho sugere que a marcha com calçados difere do deambular descalço nas curvas cinemáticas do ALM do pé, tornozelo, joelho e quadril. Essa diferença pode ocorrer em maior ou menor magnitude dependendo do tipo de calçado. As crianças apresentaram maior diferença no ALM com o CB. Além disso, as menores diferenças na cinemática de tornozelo, joelho e quadril foram observadas quando as crianças usaram calçados mais largos na região do antepé.

\section{Fontes de Suporte}

O LAM foi parcialmente financiado pela Coordenação de Aperfeiçoamento de Pessoal de Nível Superior (CAPES código de financiamento 001), e esta pesquisa foi financiada pela Anamê Ciência e Tecnologia em Saúde Infantil Ltda.

\section{Conflito de interesses}

A Dra Liria Akie Okai-Nóbrega e a Dra Ana Paula Lage trabalham com pesquisa na Anamê Ciência e Tecnologia em Saúde Infantil Ltda.

\section{Referências}

1 Kung SM, Fink PW, Hume P, Shultz SP. Kinematic and kinetic differences between barefoot and shod walking in children. Footwear Sci 2015;7(02):95-105

2 Staheli LT. Shoes for children: a review. Pediatrics 1991;88(02): 371-375

3 Morrison SC, Price C, McClymont J, Nester C. Big issues for small feet: developmental, biomechanical and clinical narratives on children's footwear. J Foot Ankle Res 2018;11(01):39

4 Wegener C, Hunt AE, Vanwanseele B, Burns J, Smith RM. Effect of children's shoes on gait: a systematic review and meta-analysis. J Foot Ankle Res 2011;4(01):3

5 Hill M, Healy A, Chockalingam N. Key concepts in children's footwear research: a scoping review focusing on therapeutic footwear. J Foot Ankle Res 2019;12(01):25

6 Cranage S, Perraton L, Bowles KA, Williams C. A comparison of young children's spatiotemporal measures of walking and running in three common types of footwear compared to bare feet. Gait Posture 2020;81:218-224

7 Sutherland DH, Olshen R, Cooper L, Woo SL. The development of mature gait. J Bone Joint Surg Am 1980;62(03):336-353

8 Adolph KE, Cole WG, Komati M, et al. How do you learn to walk? Thousands of steps and dozens of falls per day. Psychol Sci 2012; 23(11):1387-1394

9 Hallemans A, De Clercq D, Van Dongen S, Aerts P. Changes in footfunction parameters during the first 5 months after the onset of independent walking: a longitudinal follow-up study. Gait Posture 2006;23(02):142-148
10 Ledebt A, van Wieringen PCW, Savelsbergh GJP. Functional significance of foot rotation asymmetry in early walking. Infant Behav Dev 2004;27(02):163-172

11 Kelikian AS, Ed. Sarrafian's Anatomy of the Foot and Ankle: Descriptive, Topographic, Functional. 3rd ed. Philadelphia: Lippincot Williams \&Wilkins; 2011

12 Hollander K, van der Zwaard BC, de Villiers JE, Braumann KM, Venter R, Zech A. The effects of being habitually barefoot on foot mechanics and motor performance in children and adolescents aged 6-18 years: study protocol for a multicenter cross-sectional study (Barefoot LIFE project). J Foot Ankle Res 2016;9(01):36

13 Kurup HV, Clark CIM, Dega RK. Footwear and orthopaedics. Foot Ankle Surg 2012;18(02):79-83

14 Holowka NB, Wallace IJ, Lieberman DE. Foot strength and stiffness are related to footwear use in a comparison of minimally- vs. conventionally-shod populations. Sci Rep 2018;8(01):3679

15 Lejeune TM, Willems PA, Heglund NC. Mechanics and energetics of human locomotion on sand.J Exp Biol 1998;201(Pt 13): 2071-2080

16 van den Berg MEL, Barr CJ, McLoughlin JV, Crotty M. Effect of walking on sand on gait kinematics in individuals with multiple sclerosis. Mult Scler Relat Disord 2017;16:15-21

17 Stenvinkel P, Painer J, Johnson RJ, Natterson-Horowitz B. Biomimetics- Nature's roadmap to insights and solutions for burden of lifestyle diseases. Journal of Internal Medicine 2020;287(03): 238-251

18 Portney LG, Watkins MP. The role of theory in clinical research. In: Foundations of Clinical Research: Applications to Practice. Connecticut: Appleton \& Lange; 1993:17-26

19 Robertson GE, Caldwell GE, Hamill J, Kamen G, Whittlesey SN Research Methods in Biomechanics. Champaign, IL: Human Kinetics; 2014

20 Heyrman L, Feys H, Molenaers G, et al. Three-dimensional head and trunk movement characteristics during gait in children with spastic diplegia. Gait Posture 2013;38(04):770-776

21 Michael W, Richard S, Lee S. Feet and Footwear: Applying Biological Design and Mismatch Theory to Running Injuries. Int J Sport Exerc Med 2018;4(02):1-7

22 Bencke J, Christiansen D, Jensen K, Okholm A, Sonne-Holm S, Bandholm T. Measuring medial longitudinal arch deformation during gait. A reliability study. Gait Posture 2012;35(03):400-404

23 Bandholm T, Boysen L, Haugaard S, Zebis MK, Bencke J. Foot medial longitudinal-arch deformation during quiet standing and gait in subjects with medial tibial stress syndrome. J Foot Ankle Surg 2008;47(02):89-95

24 Gomes RBO, Souza TR, Paes BDC, et al. Foot pronation during walking is associated to the mechanical resistance of the midfoot joint complex. Gait Posture 2019;70:20-23

25 Onodera AN, Sacco IC, Morioka EH, Souza PS, de Sá MR, Amadio AC. What is the best method for child longitudinal plantar arch assessment and when does arch maturation occur? Foot 2008;18 (03):142-149

26 Price C, McClymont J, Hashmi F, Morrison SC, Nester C. Development of the infant foot as a load bearing structure: study protocol for a longitudinal evaluation (the Small Steps study). J Foot Ankle Res 2018;11:33

27 Thelen E. Motor development. A new synthesis. Am Psychol 1995; 50(02):79-95

28 Franklin S, Grey MJ, Heneghan N, Bowen L, Li FX. Barefoot vs common footwear: A systematic review of the kinematic, kinetic and muscle activity differences during walking. Gait Posture 2015;42(03):230-239

29 Neumann DA. Kinesiology of the Musculoskeletal System: Foundations for Rehabilitation. 2nd. London: Mosby; 2009

30 Souza TR, Pinto RZ, Trede RG, Kirkwood RN, Fonseca ST. Temporal couplings between rearfoot-shank complex and hip joint during walking. Clin Biomech (Bristol, Avon) 2010;25(07):745-748 